\title{
Screening potential biomarkers for colorectal cancer based on circular RNA chips
}

\author{
SHUO CHEN, LIN ZHANG, YINAN SU and XIPENG ZHANG \\ Department of Anal and Intestinal Surgery, Tianjin Union Medical Center \\ (Nankai University Affiliated Hospital), Tianjin 300000, P.R. China
}

Received October 25, 2017; Accepted March 12, 2018

DOI: $10.3892 /$ or.2018.6372

\begin{abstract}
The aim of the present study was to screen colorectal cancer (CRC) tissue and adjacent tissue for differences in circular RNA (circRNA) expression, to analyze the related miRNAs and messenger RNAs (mRNAs), and to investigate the circRNA expression in CRC and its function. The circRNA expression profile was generated using CapitalBio microarray technology. The differentially expressed circRNAs were identified with GeneSpring 12.5 software. Subsequently, the related mRNAs of the differentially expressed circRNAs were annotated with the molecule annotation system (MAS) 3.0, and the diseases, pathways and functional enrichment analysis of these mRNAs were performed using the KEGG orthology-based annotation system (KOBAS) 3.0. In addition, the target miRNAs of differentially expressed circRNAs were screened using the miRanda algorithm. The circRNA/miRNA network was constructed for the top 8 most significant differentially expressed circRNAs with Cytoscape software 3.4.0. A total of 10,245 differentially expressed circRNAs were identified, including 6,264 upregulated ones, and 3,981 downregulated ones. The related $\mathrm{mRNAs}$ were enriched in $462 \mathrm{KEGG}$ diseases, 411 FunDO, 669 NHGRI GWAS catalog, and 845 OMIM; and 1,334 Reactomes, 281 KEGG pathways, 117 PANTHER and 193 BioCyc; and 11,606 Gene Ontology (GO) terms. A total of 133 circRNA/miRNA pairs were involved in the circRNA/miRNA network. $h s a \_c i r c \_0126897 \_C B C 1$ may be a potential biomarker for CRC, and the cell cycle was closely associated with the occurrence and development of CRC.
\end{abstract}

\section{Introduction}

Colorectal cancer (CRC) ranks third in terms of high incidence and as the fourth leading cause of cancer-related deaths

Correspondence to: Dr Xipeng Zhang, Department of Anal and Intestinal Surgery, Tianjin Union Medical Center (Nankai University Affiliated Hospital), 190 Jie-Yuan Road, Hongqiao, Tianjin 300000, P.R. China

E-mail: chenshuo19801106@126.com

Key words: circular RNAs, colorectal cancer, KEGG orthology based annotation system, circular RNA-miRNA network worldwide (1). It is estimated that $\sim 1.3$ million new cases are diagnosed and nearly 0.7 million succumb to CRC each year worldwide (1). CRC incidence rates are highest in Alaska Natives and African Americans and lowest in Asian/Pacific Islanders, and they are 30-40\% higher in men than in women (2). The incidence and mortality of CRC increase with age, and $\sim 50 \%$ of CRC patients are older than 70 years (1). Currently, screening is one of the most effective diagnostic methods, and treatments mainly include surgery, chemotherapy, radiation therapy, targeted therapy and some combination therapy. The incidence and death rates have declined in both men and women in recent years, as a result of improved screening and advances in clinical treatment (1). However, the potential mechanism of $\mathrm{CRC}$ has not been clarified clearly, especially at the molecular level (3).

Circular RNAs (circRNAs), small ( 21 nt) non-coding RNAs, that form a covalently closed continuous loop, are characterized by high expression levels, stability, and a large number of miRNA binding sites (4). Due to the fact that circRNAs do not have $5^{\prime}$ or $3^{\prime}$ ends, they are resistant to exonuclease-mediated degradation and share high evolutionary conservation in cells (5). They base-pair directly target messenger RNAs (mRNAs), and can trigger cleavage of the mRNA depending on the degree of complementarity. circRNAs are considered to be involved in a large, diverse set of biological processes (BPs), such as gene regulation (6). Research has demonstrated that some circRNAs are associated with the bioprocesses of proliferation, metastasis, prognosis and a curative effect in CRC (1).

At present, the biological functions of most circRNAs are unclear, and little is known about circRNAs and their relationship with CRC. It is worthwhile to examine how circRNAs can be used to study the pathogenesis and some BPs of CRC. In the present study, the differentially expressed circRNAs, as well as their related mRNAs and miRNAs, were screened and analyzed in CRC tissue compared with adjacent tissue, in order to explore the potential molecular mechanism from the circRNA expression levels.

\section{Materials and methods}

Patients and sample collection. Four samples from CRC patients, aged 50 to 62 years, were collected from Tianjin Union Medical Center, Nankai University Affiliated Hospital 
in 2016. The patients had not been treated with radiotherapy or chemotherapy before the surgery. The CRC tissue and their adjacent tissue samples were obtained after CRC surgeries, and recorded as treatment and control groups, respectively. The experimental study was approved by the hospital ethics committee, and the informed consent forms were obtained when the patients were accepted for the study by the hospital. All procedures performed in the studies involving human participants were in accordance with the ethical standards of Committee of the Tianjin Union Medical Center (Tianjin, China) and with the 1964 Helsinki declaration and its later amendments or comparable ethical standards.

Sample processing and circRNA acquisition. The samples from the two groups were immediately washed with $0.9 \% \mathrm{NaCl}$ (RNase-free) and quickly dipped in RNase inhibitor (Epicentre: Illumina, Inc., San Diego, CA, USA) according to the manufacturer's instructions. Then, total RNA was extracted from the CRC tissues using TRIzol (Invitrogen: Thermo Fisher Scientific, Inc., Waltham, MA, USA) according to the manufacturer's instructions. Total RNA was digested with RNase R (Epicentre: Illumina, Inc.) to remove linear RNAs, and was then reversed transcribed as cDNA. The cDNA was purified with NucleoSpin ${ }^{\circledR}$ Extract II kits (Macherey-nagel, Düren, Germany), and was labeled with a fluorescent probe (Arraystar Super RNA Labeling kit; Arraystar, Inc., Rockville, MD, USA). The fluorescent cRNA was purified with the the NucleoSpin ${ }^{\circledR}$ Extract II kits. The concentration and specific activity of the labeled cRNAs (pmol Cy3/ $\mu \mathrm{g}$ cRNA) were assessed using NanoDrop ND-1000 (Thermo Fisher Scientific, Inc.). A total of $1 \mu \mathrm{g}$ of each labeled cRNA was fragmented by adding $5 \mu \mathrm{l}$ of $10 \mathrm{X}$ blocking agent and $1 \mu \mathrm{l}$ of $25 \mathrm{X}$ fragmentation buffer. Finally, $25 \mu 1$ of $2 \mathrm{X}$ hybridization buffer was added to dilute the labeled cRNA. Then, a total of $50 \mu 1$ mixtures were heated at $95^{\circ} \mathrm{C}$ for $3 \mathrm{~min}$, and cooled $2 \mathrm{~min}$ on ice. The hybridization solution was dispensed into the gasket slide and assembled to construct the circRNA expression microarray slide. The slides were centrifuged for $16 \mathrm{~h}$ at $40 \mathrm{x} \mathrm{g}$ at $65^{\circ} \mathrm{C}$ in an Agilent Hybridization Oven (Agilent Technologies, Inc., Santa Clara, CA, USA). Finally, the hybridized arrays were washed, fixed and scanned using the Agilent scanner G2565CA (Agilent Technologies, Inc.).

Single array analysis and cluster analysis. The Agilent Feature Extraction 10.7 software (Agilent Technologies, Inc.) graph chip was used to read the values and to obtain the original data. The GeneSpring GX 12.5 software (Agilent Technologies, Inc.) was used for quintile normalization and subsequent data processing of the original data. After standardization, the cluster analyses of the eight samples were performed with hierarchical and average linkage algorithms of R package.

Identification of the differential expressed circRNAs. After single array and cluster analysis, the differentially expressed circRNAs were identified in CRC tissue samples compared with the adjacent tissue samples using $\mathrm{P}<0.05$ and $\mid \log 2$ (fold-change) $\mid>1$. Moreover, the volcano plot was plotted according to the P-value and $\log 2$ (fold change). In addition, the Circos plot also was drawn.
Screening related $m R N A s$ and enrichment analysis. KEGG orthology based annotation system (KOBAS) is a web server for gene/protein functional annotation (annotate module) and functional gene set enrichment (enrichment module) (1). In the present study, the related mRNAs of the differentially expressed circRNAs were annotated with the molecule annotation system (MAS) 3.0, and the diseases, pathways and functional enrichment analysis, in which they were enriched, were performed using KOBAS 3.0. The terms of enriched diseases included KEGG disease, FunDO, NHGRI GWAS catalog and OMIM; the enriched pathways contained Reactome, KEGG pathways, PANTHER and BioCyc; and BPs, molecular function (MF) and cellular component (CC) were involved in the Gene Ontology (GO) terms.

Screening related target miRNAs and construction of the circRNA/miRNA network. A circRNA can bind to a miRNA and indirectly regulate the translation of an mRNA, and miRanda is an algorithm used to find genomic targets for miRNAs. The related target miRNAs for the differentially expressed circRNAs were predicted with the miRanda algorithm. Subsequently, the key circRNAs with high $\log 2$ (fold-change)I, which were involved in the most circRNA/miRNA pairs were chosen. Ultimately, the circRNA/miRNA network for the key circRNAs was constructed and visualized using Cytoscape (7) software (Cytoscape 3.4.0, http://www.cytoscape.org/).

Verification of related circRNAs and its target genes. The tumor tissues and paracancerous specimens from four CRC patients were collected, after the patients had signed informed consent. The target gene of hsa_circ_0126897_CBCl was found to be SLC4A4 from circBase (http://circrna. org/cgi-bin/singlerecord.cgi?id=hsa_circ_0126897). The SYBR $^{\circledR}$ Premix Ex Taq ${ }^{\mathrm{TM}}$ kit (Takara Biotechnology Co., Ltd., Shiga, Japan) and the Applied Biosystems ${ }^{\mathrm{TM}}$ QuantStudio $^{\mathrm{TM}}$ 5 Real-Time PCR System (Applied Biosystems; Thermo Fisher Scientific, Foster City, CA, USA) were used to detect the mRNA levels of hsa_circ_0126897_CBCl and SLC4A4 according to the manufacturer's instructions. All the primers were designed and synthesized by Takara Biotechnology Co., Ltd. (Beijing, China). The primers of hsa_circ_0126897_ CBC1: 3'-CTCATGGTGATGCTGAACCTTCTTA-5' and 3'-CTCATGGTGATGCTGAACCTTCTTA-5' (139 bp); and SLC4A4: 3'-AGCACTCTATACGCCCCAAG-5' and 3'-TTC CTTTTCTCTCACGCCCT-5' (540 bp). In addition, $\beta$-actin was used as a reference, and the primer sequences were $5{ }^{\prime}-\mathrm{CTA}$ CAATGAGCTGCGTGTGG-3' and 5'-AGGCATACAGGG ACAACACA-3' (308 bp).

Statistical analysis. SPSS v17.0 (SPSS, Inc., Chicago, IL, USA) was used for all statistical analyses, and data were expressed as the mean $\pm \mathrm{SD}$. To compare both two groups $\mathrm{t}$-test was used, and $\mathrm{P}<0.05$ was considered statistically significant.

\section{Results}

Differentially expressed circRNAs. After single array analysis, the cluster analysis between the two groups was performed. The cluster graph is displayed in Fig. 1A, which revealed that 4 CRC 
A
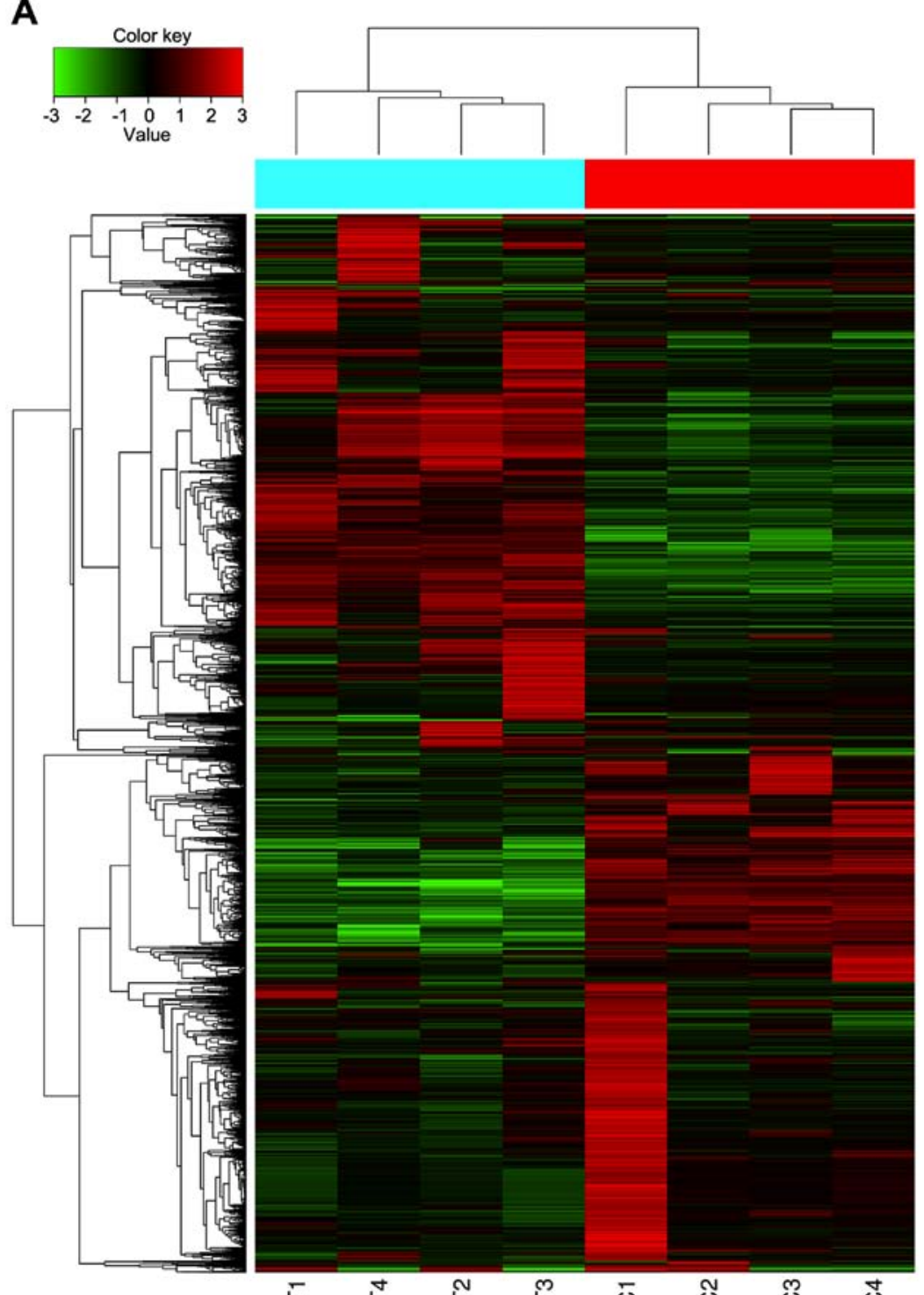

F
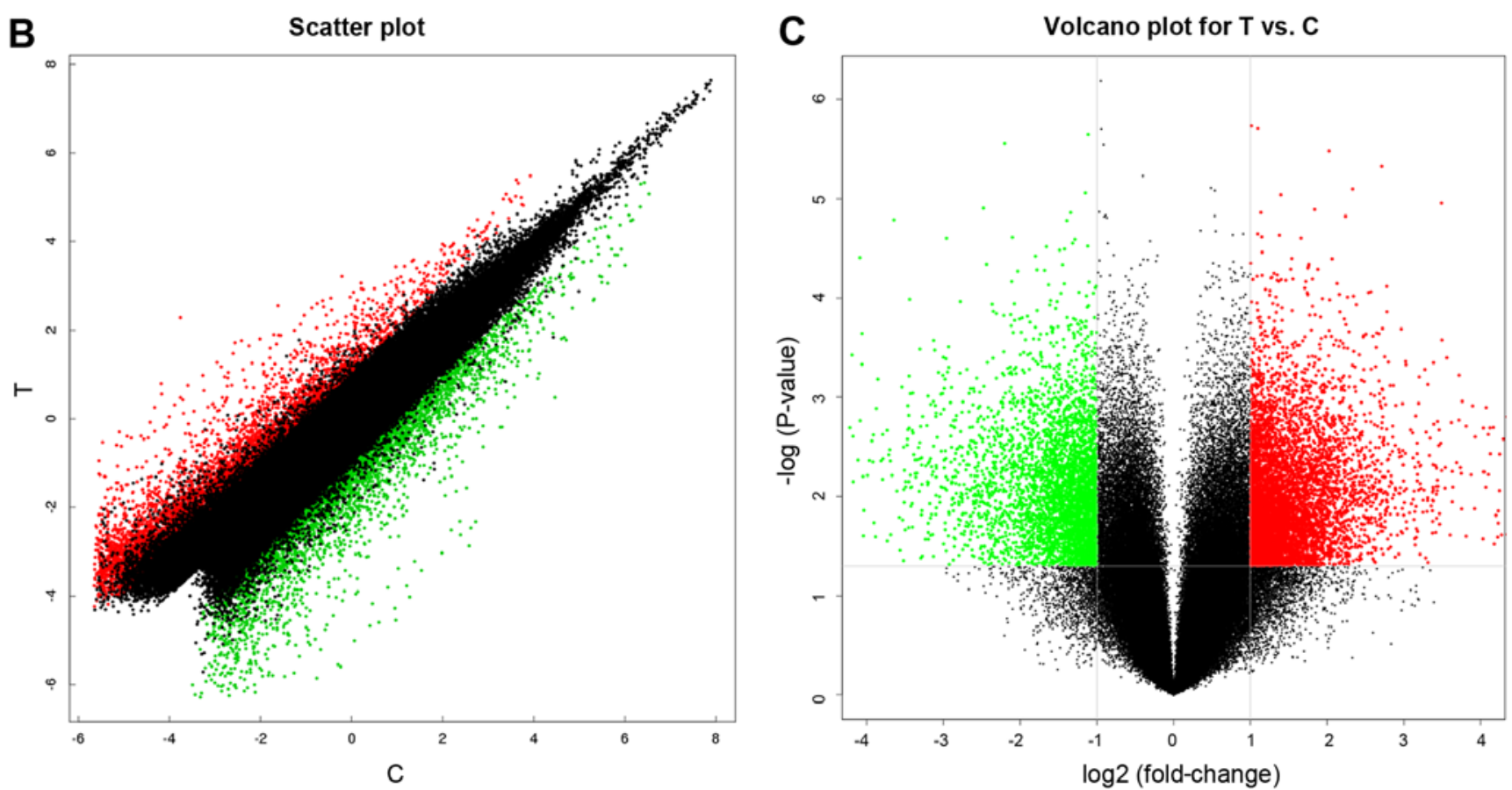

Figure 1. Identification of the differentially expressed circRNAs. (A) The cluster graph of the samples, (B) the scatter plot and (C) the volcano plot. 


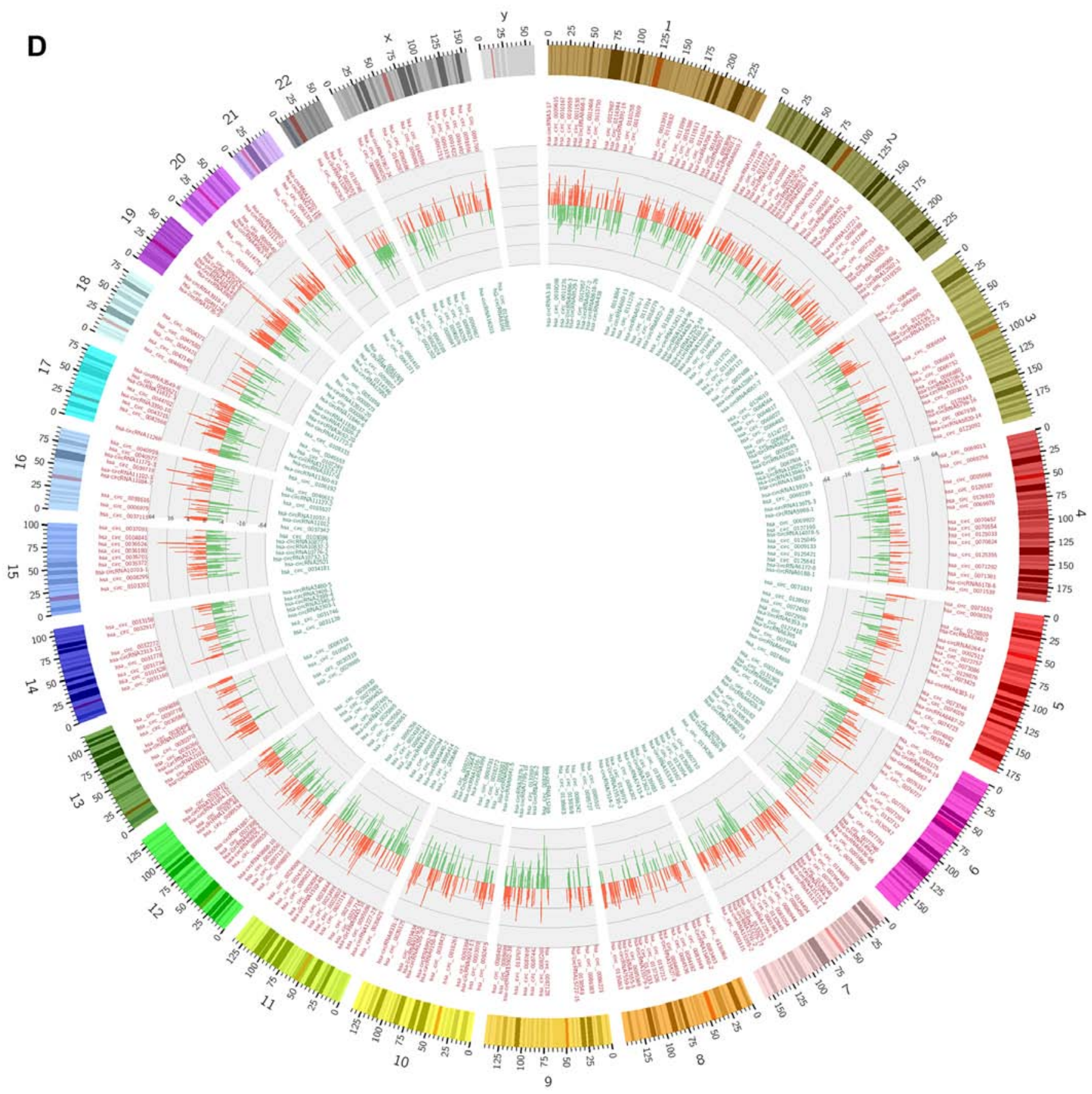

Figure 1. Continued. (D) the Circos plot. circRNA, circular RNA.

tissue samples (T1-T4) were clustered into one group, and the 4 adjacent tissue samples (C1-C4) were clustered into one class. Differentially expressed circRNAs were identified according to fluorescence signal intensity (Fig. 1B), and the volcano plot and the Circos plot are presented in Fig. 1C and D, respectively. Fig. 1 revealed that the differentially expressed circRNAs could separate tumor samples from paracancerous tissues, commendably. A total of 10,245 (6,264 up- and 3,981 downregulated) differentially expressed circRNAs were identified, and the top 30 most significant ones are presented in Table I. It was evident from Table I that hsa_circ_0027997_CBC1, $h s a+c i r c \_0060967 \_C B C 1$ and $h s a+c i r c \_0055546 \_C B C 1$ were the top 3 differentially expressed circRNAs.
The related $m R N A s$ and enriched terms. The related mRNAs of the differentially expressed circRNAs were annotated by KOBAS 3.0, and they were enriched in some disease terms, GO terms and pathways. Furthermore, the enriched diseases included 462 KEGG diseases, 411 FunDO, 669 NHGRI GWAS catalog, and 845 OMIM, and the top 30 disease terms are shown in Fig. 2A-D. The KEGG diseases contained breast and ovarian cancer as well as CRC. The FunDO contained skin and stomach cancer as well as adenocarcinoma The NHGRI GWAS catalog contained lung and bladder cancer, and OMIM contained glioblastoma, gastric cancer and CRC. The enriched GO terms contained 8,485 BP; 2,026 MF; and 1,095 CC, and their hierarchy and top $30 \mathrm{GO}$ terms are shown in Fig. 3A-D. The cell 
A

Significant enriched KEGG DISEASES. Disease terms (top 30)

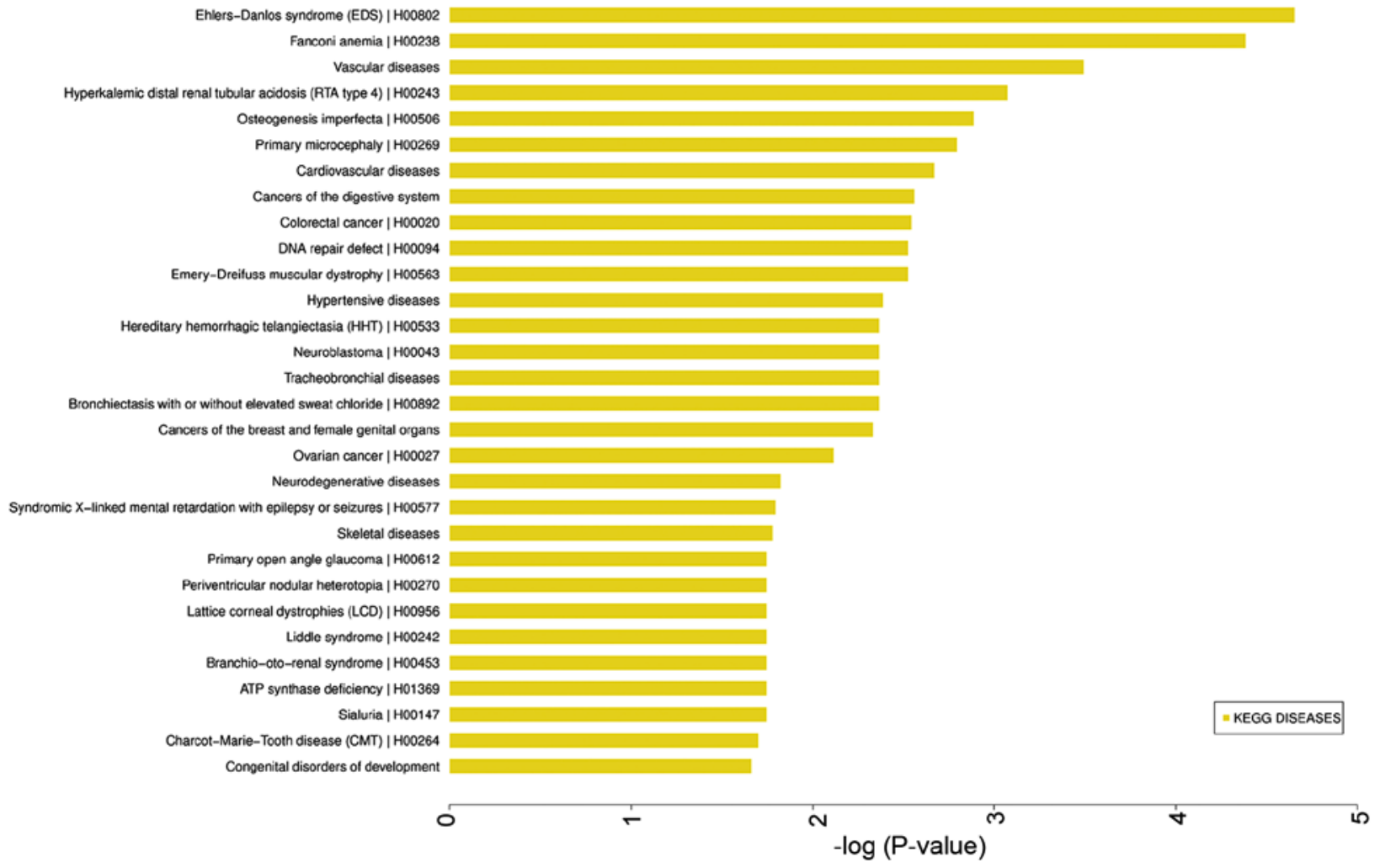

B Significant enriched FunDO. Disease terms (top 30)

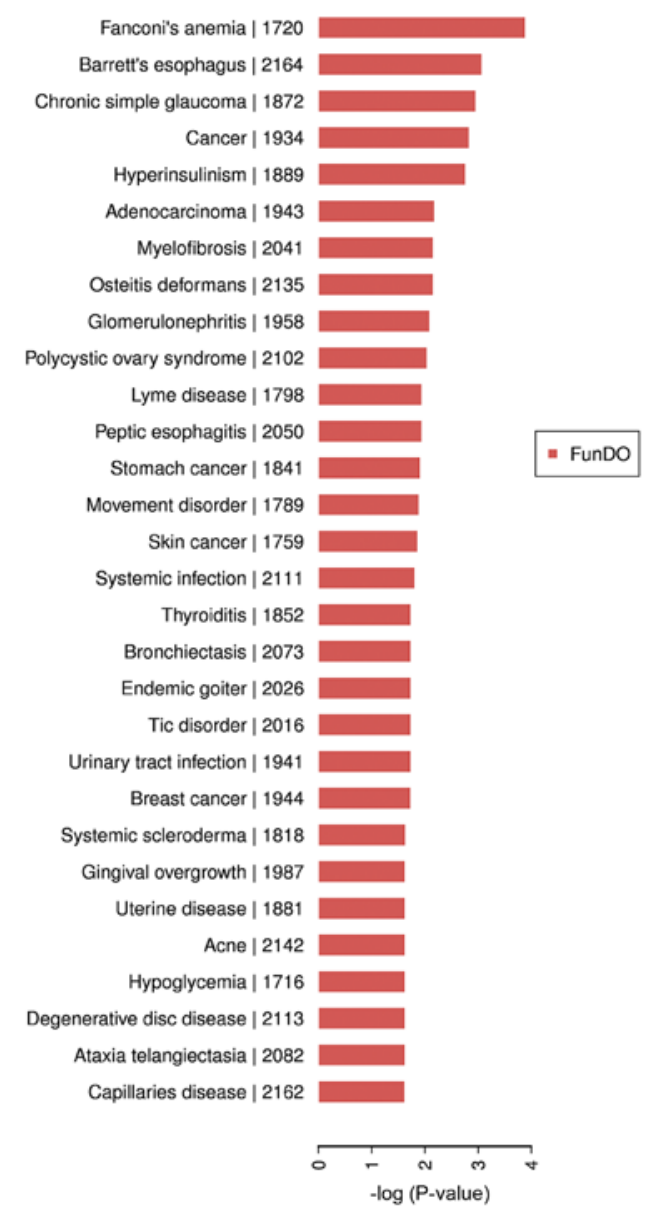

Figure 2. The enriched diseased terms. The top 30 most significant enriched (A) KEGG diseases terms and (B) FunDO terms. 


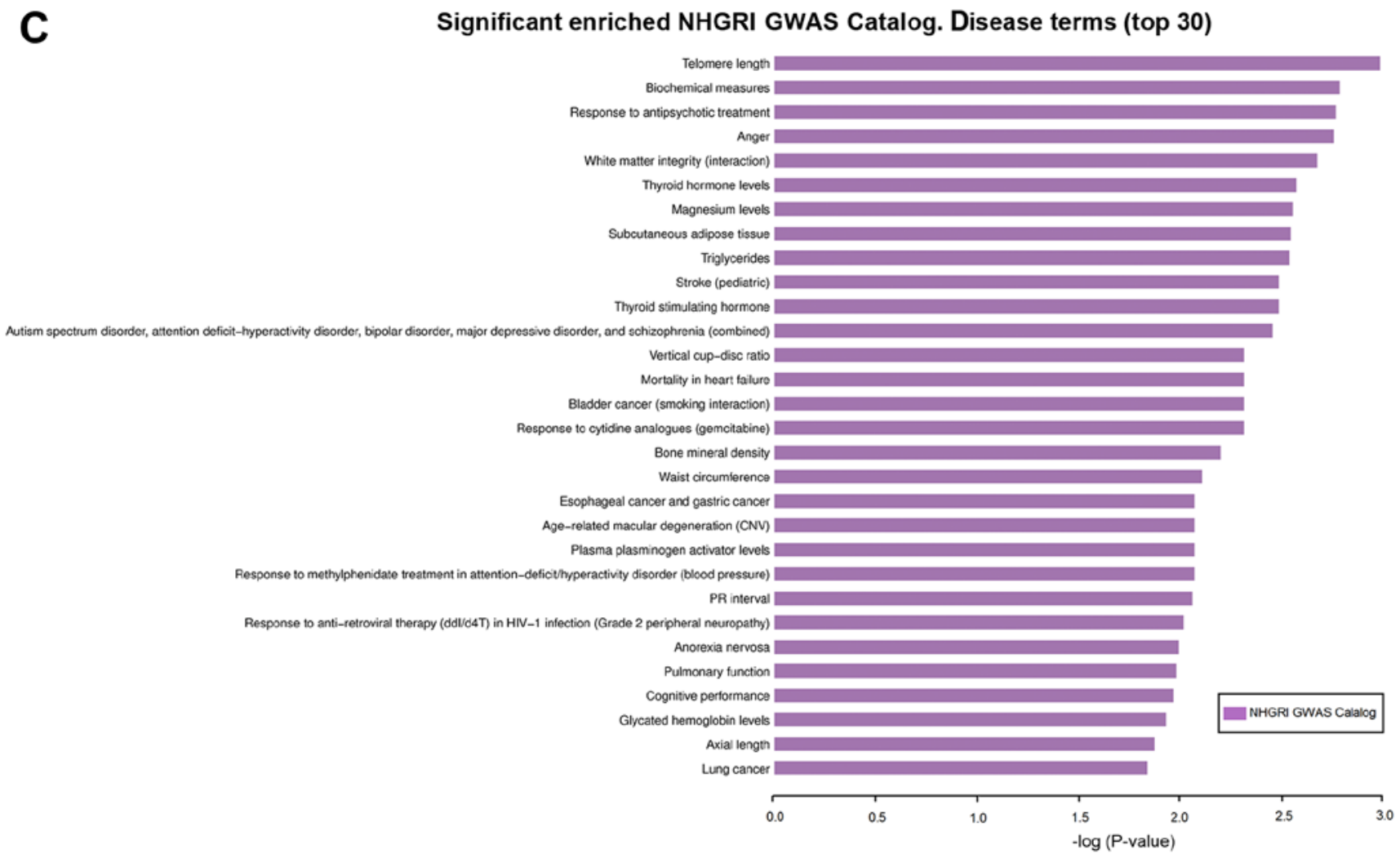

D

Significant enriched OMIM. Disease terms (top 30)

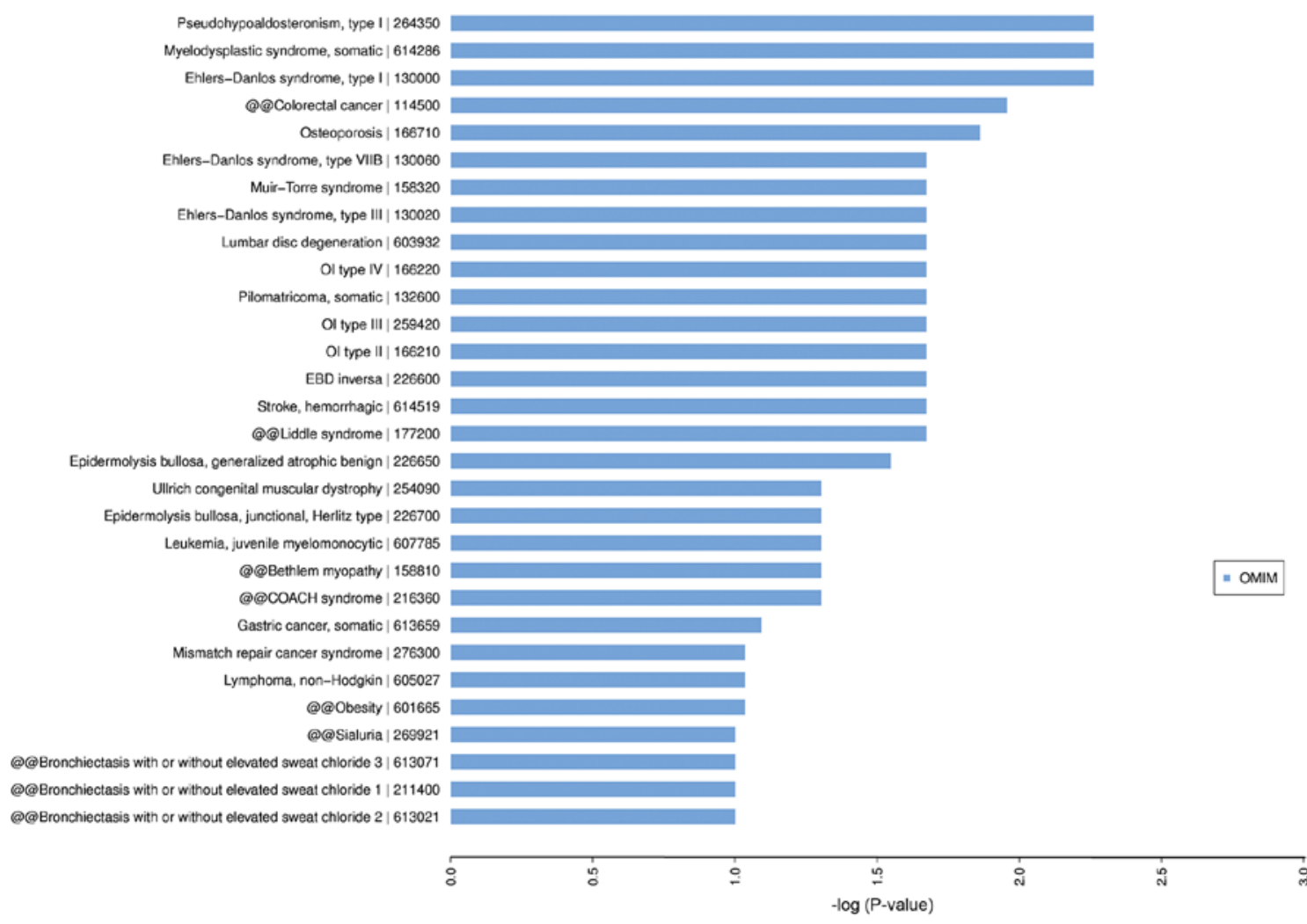

Figure 2. Continued. The top 30 most significant enriched (C) HGRI GWAS catalog terms and (D) OMIM terms.

cycle process, the mitotic cell cycle and the cell cycle were the top 3 most significant BPs (Fig. 3D). A total of 1,334 Reactomes, 281 KEGG pathways, 117 PANTHER and 193 BioCyc were involved in the enriched pathways, and the top 30 terms of each are shown in Fig. 4A-D. Cell cycle, and 'cell cycle, mitotic' were the top 2 most significant Reactome pathways, cell cycle was the most significant KEGG pathway, and DNA replication was the most significant PANTHER pathway. 
Table I. The top 30 most significant differentially expressed circRNAs based on the P-value.

\begin{tabular}{|c|c|c|c|}
\hline circRNAs name & P-value & $\mathrm{FC}$ & Regulation \\
\hline hsa_circ_0027997_CBC1 & 0.000307 & 2.402326 & Down \\
\hline hsa_circ_0060967_CBC1 & 0.000315 & 2.264531 & Up \\
\hline hsa_circ_0055546_CBC1 & 0.000344 & 2.086458 & Down \\
\hline hsa-circRNA7788-9_CBC1 & 0.000514 & 2.821571 & Down \\
\hline hsa_circ_0004619_CBC1 & 0.00053 & 2.251397 & Down \\
\hline hsa-circRNA5955-2_CBC1 & 0.000618 & 2.216281 & Down \\
\hline hsa_circ_0089626_CBC1 & 0.000743 & 2.603937 & Down \\
\hline hsa_circ_0047851_CBC1 & 0.000887 & 2.100605 & Down \\
\hline hsa_circ_0109686_CBC1 & 0.001066 & 3.009866 & Down \\
\hline hsa_circ_0026344_CBC1 & 0.001555 & 5.709074 & Down \\
\hline hsa_circ_0073207_CBC1 & 0.001635 & 2.687123 & Down \\
\hline hsa_circ_0088103_CBC1 & 0.001688 & 2.40819 & Down \\
\hline hsa_circ_0094226_CBC1 & 0.001766 & 5.875593 & Down \\
\hline hsa_circ_0070283_CBC1 & 0.001785 & 4.989792 & Down \\
\hline hsa_circ_0103088_CBC1 & 0.001941 & 2.771014 & Down \\
\hline hsa_circ_0136666_CBC1 & 0.002348 & 3.634452 & Up \\
\hline hsa-circRNA1102_CBC1 & 0.00248 & 3.036569 & Down \\
\hline hsa-circRNA7032-14_CBC1 & 0.002612 & 2.136622 & $\mathrm{Up}$ \\
\hline hsa-circRNA6158-6_CBC1 & 0.002704 & 3.008672 & Down \\
\hline hsa_circ_0011276_CBC1 & 0.00276 & 2.926497 & Down \\
\hline hsa_circ_0031263_CBC1 & 0.003919 & 7.843025 & Up \\
\hline hsa_circ_0069013_CBC1 & 0.004342 & 2.26783 & $\mathrm{Up}$ \\
\hline hsa_circ_0011924_CBC1 & 0.004687 & 3.432366 & $\mathrm{Up}$ \\
\hline hsa-circRNA3677-10_CBC1 & 0.004807 & 2.059462 & Down \\
\hline hsa_circ_0005525_CBC1 & 0.005082 & 2.523352 & Down \\
\hline hsa_circ_0060745_CBC1 & 0.005263 & 2.027748 & Up \\
\hline hsa_circ_0042328_CBC1 & 0.005733 & 2.532399 & Down \\
\hline hsa_circ_0072715_CBC1 & 0.005974 & 2.193656 & Up \\
\hline hsa_circ_0014712_CBC1 & 0.006073 & 2.107837 & Up \\
\hline hsa-circRNA3698-20_CBC1 & 0.006194 & 2.07261 & Down \\
\hline
\end{tabular}

circRNAs, circular RNAs.

CircRNA/miRNA network. The related target miRNAs for the differentially expressed circRNAs were predicted. Then, the eight key differentially expressed circRNAs, which were associated with the most target miRNAs were chosen. They

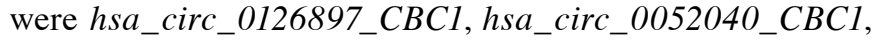
hsa-circRNA6015-4_CBC1, hsa_circ_0126905_CBC1, hsa-circRNA15787_CBC1, hsa_circ_0138971_CBC1, $h s a+c i r c \_0126905 \_C B C 1$ and $h s a-c i r c R N A 9432 \_C B C 1$, and were involved in 133 circRNA/miRNA pairs. Finally, the circRNA/miRNA network was established, and is presented in Fig.5.In the network, $h s a \_c i r c \_0126897 \_C B C l$ was involved in the most pairs, which was significantly downregulated in the CRC samples compared with their adjacent tissue samples. In addition, the scatter plot figure of $h s a \_c i r c \_0126897 \_C B C 1$ and its target gene (SLC4A4) is presented in Fig. 6. The mRNA level of $h s a \_c i r c \_0126897 \_C B C 1$ was significantly decreased in the CRC samples compared to their adjacent tissue samples
$(\mathrm{P}<0.0001, \mathrm{t}=24.70)$. Moreover, the expression of target gene (SLC4A4) was also decreased in the CRC samples $(\mathrm{P}<0.0001$, $\mathrm{t}=13.72)$.

\section{Discussion}

circRNAs can act as competing endogenous RNAs or miRNA sponges, regulating alternative splicing or transcription, modulating the expression of the parental gene, managing the local concentration of RBPs and playing an important role in oncogenesis and the malignant behavior of cancer (8). Recent studies have reported that a global reduction of circRNA abundance was observed in CRC samples compared with healthy tissue, therefore allowing for the proliferation of CRC cells $(9,10)$. In the present study, we not only found many downregulated circRNAs but also many upregulated ones. However, the related molecular mechanism was complex and associated 


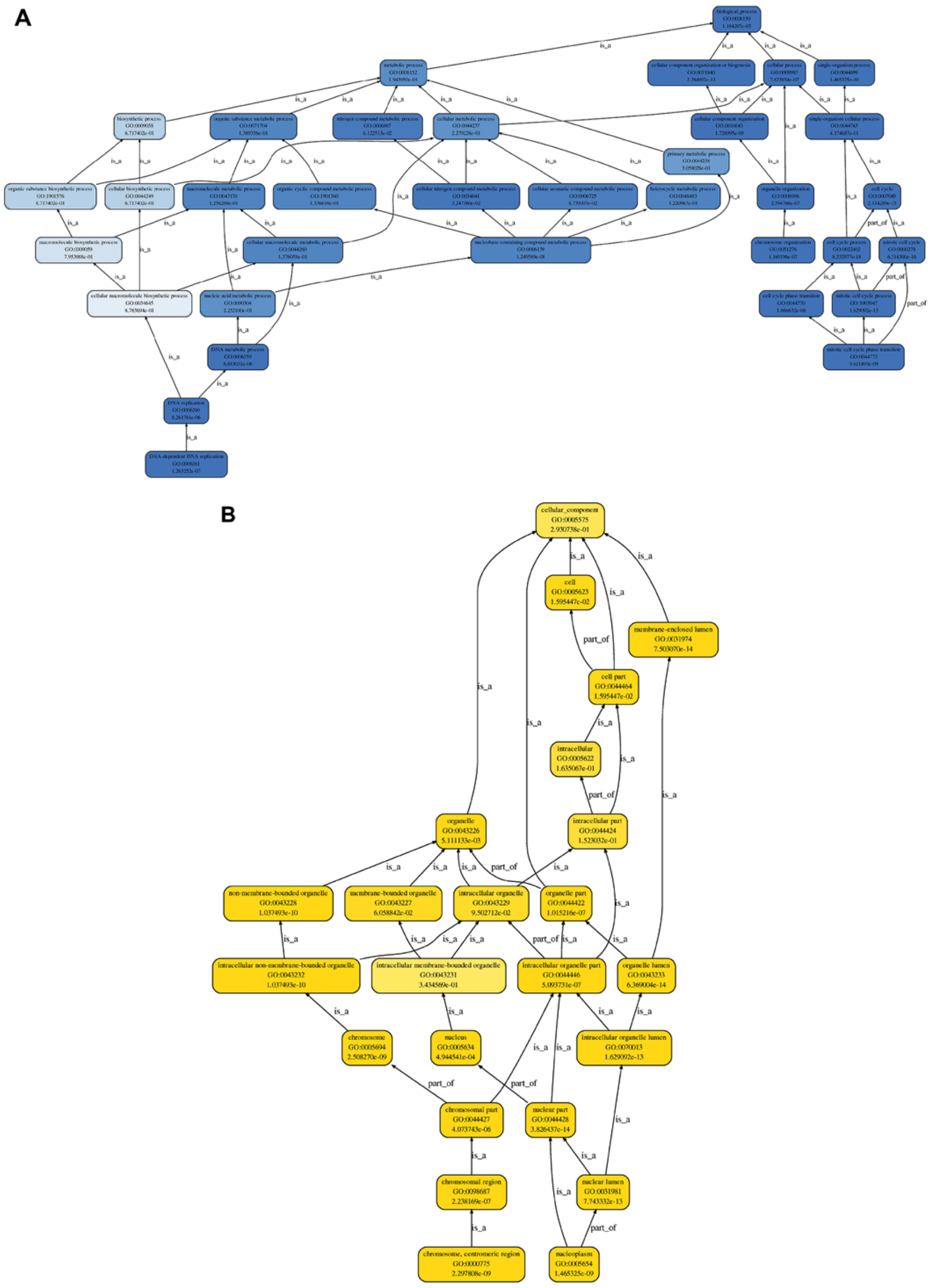

Figure 3. The enriched GO terms. The hierarchy of enriched (A) BP and (B) MF. 


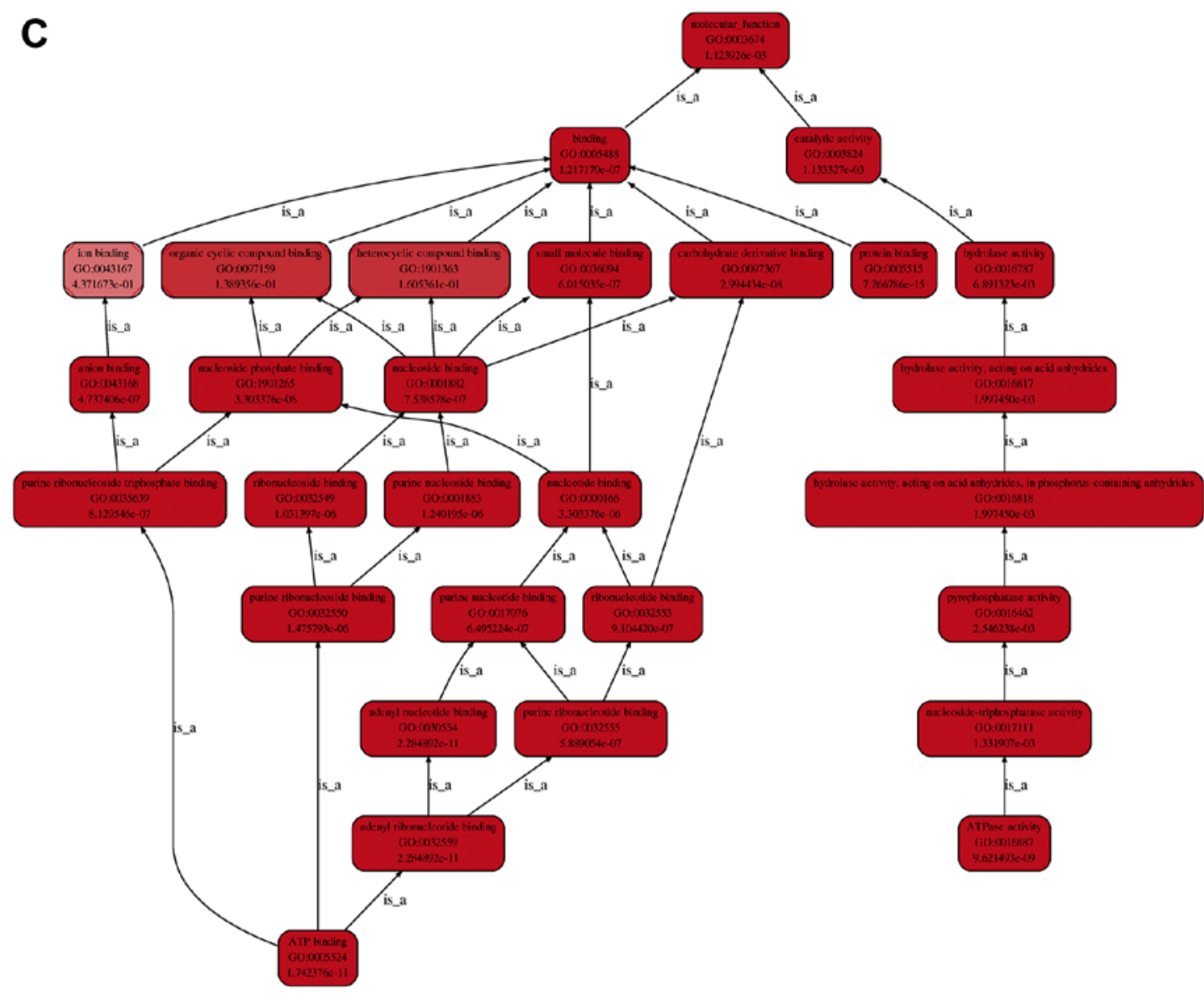

Figure 3. Continued. (C) The hierarchy of enriched CC.

with mRNA, miRNAs, protein translation, and regulation of some GO terms and pathways. Therefore, the related mRNAs and miRNAs were screened.

The enriched GO term results revealed that the top 3 BB terms were cell cycle, mitotic cell cycle and cell cycle process (Fig. 3A), which were BPs associated with the cell cycle. The cell cycle is a highly organized process regulated by cyclins, cyclin-dependent kinases (CDKs), and cyclin-dependent kinase inhibitors. Recent studies suggested that altered expression of cyclins may be involved in human cancer proliferation and development $(11,12)$. CRC could be controlled by intervening in the cell cycle, such as targeting cyclin G (13). At present, only a few studies have reported that circRNAs were involved in the BPs of the cell cycle. A recent study demonstrated that the circRNA circ-Foxo3 was highly expressed in non-cancer cells, and suppressed cell cycle progression by binding to CDK1 and CDK2 (14). Another

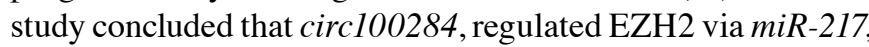
and was involved in the arsenite-accelerated cell cycle of human keratinocytes in carcinogenesis (15). Therefore, we suspected that circRNAs may regulate some mRNAs, which were associated with the cell cycle, thus having an effect on CRC. Moreover, the top 2 reactome pathways were cell cycle, mitotic' and 'cell cycle' (Fig. 4A), and the most significant enriched KEGG pathway was 'cell cycle' (Fig. 4B). These results further demonstrated the aforementioned views, and that the cell cycle was closely associated with the occurrence and development of CRC.
Upon completion of this study, the circRNA/miRNA network was constructed, and $h s a \_c i r c \_0126897 \_C B C 1$ was the node involved in the most circRNA/miRNA pairs (Fig. 5). Currently, some circRNAs have been identified to be related to the occurrence and development of CRC, such as $h s a_{-}$ circ_001988,hsa_circ_001569 and hsa_circ_0000069 (1). More circRNAs were considered to be biomarkers for some diseases. Qin et al (16) suspected that hsa_circ_0001649 may serve as a novel potential biomarker for HCC and function in the tumorigenesis and metastasis of HCC. Peripheral blood circRNA hsa_circ_0124644 can be used as a diagnostic biomarker of coronary artery disease (17). $h s a \_c i r c R N A \_103636$ was a potential novel diagnostic and therapeutic biomarker in major depressive disorders (18). circPVT1 may be a proliferative factor and prognostic marker in gastric cancer (19). hsa_circ_0005075 functioned as a potential biomarker in hepatocellular carcinoma development (20). However, the study of circRNAs is lacking basic research and is not as developed as mRNA and miRNA reseach. At present, no study has been retrieved, reporting the functions of $h s a_{-} c i r c \_0126897 \_C B C 1$. Furthermore, SLC4A4 is the target gene of hsa_circ_0126897_CBC1, and a $\mathrm{Na}(+) / \mathrm{HCO}_{3}(-)$ co-transporter. Parks and Pouyssegur reported that $S L C 4 A 4$ knockdown had a strong impact on cell proliferation, migration, and invasion of CRC and breast cancer cells, and also altered the expression of other proteins including CAIX (21). Hence, their results indicated that SLC4A4 contributes to cell migration and tumor cell 
D

Significant enriched GO terms (top 30)

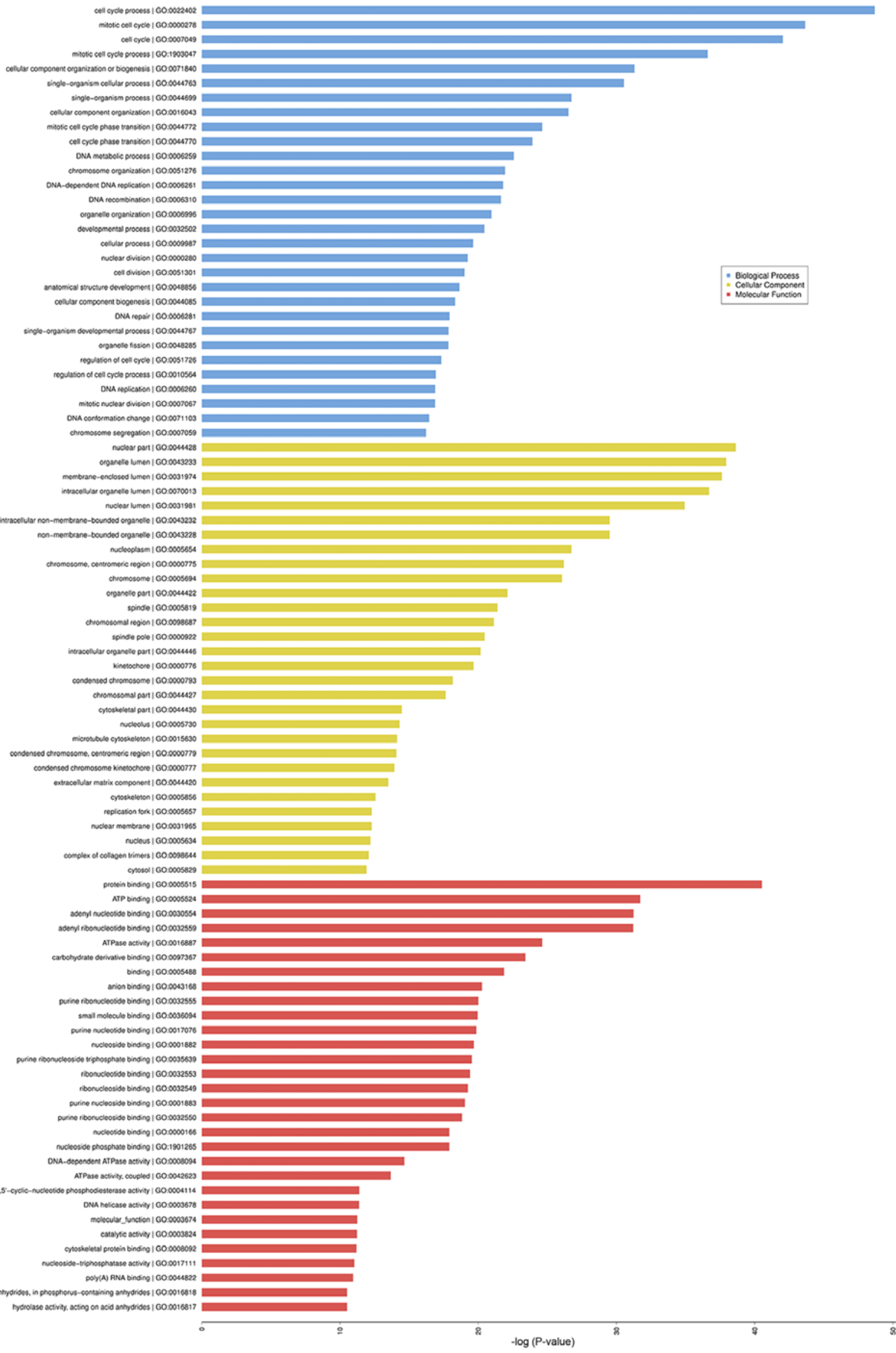

Figure 3. Continued. (D) The top 30 most significant enriched BPs (blue), MFs (yellow), and CCs (red). GO, Gene Ontology; BPs, biological processes; MF, molecular functions; $\mathrm{CC}$, cellular components. 
A

Significant enriched Reactomes. Pathway terms (top 30)

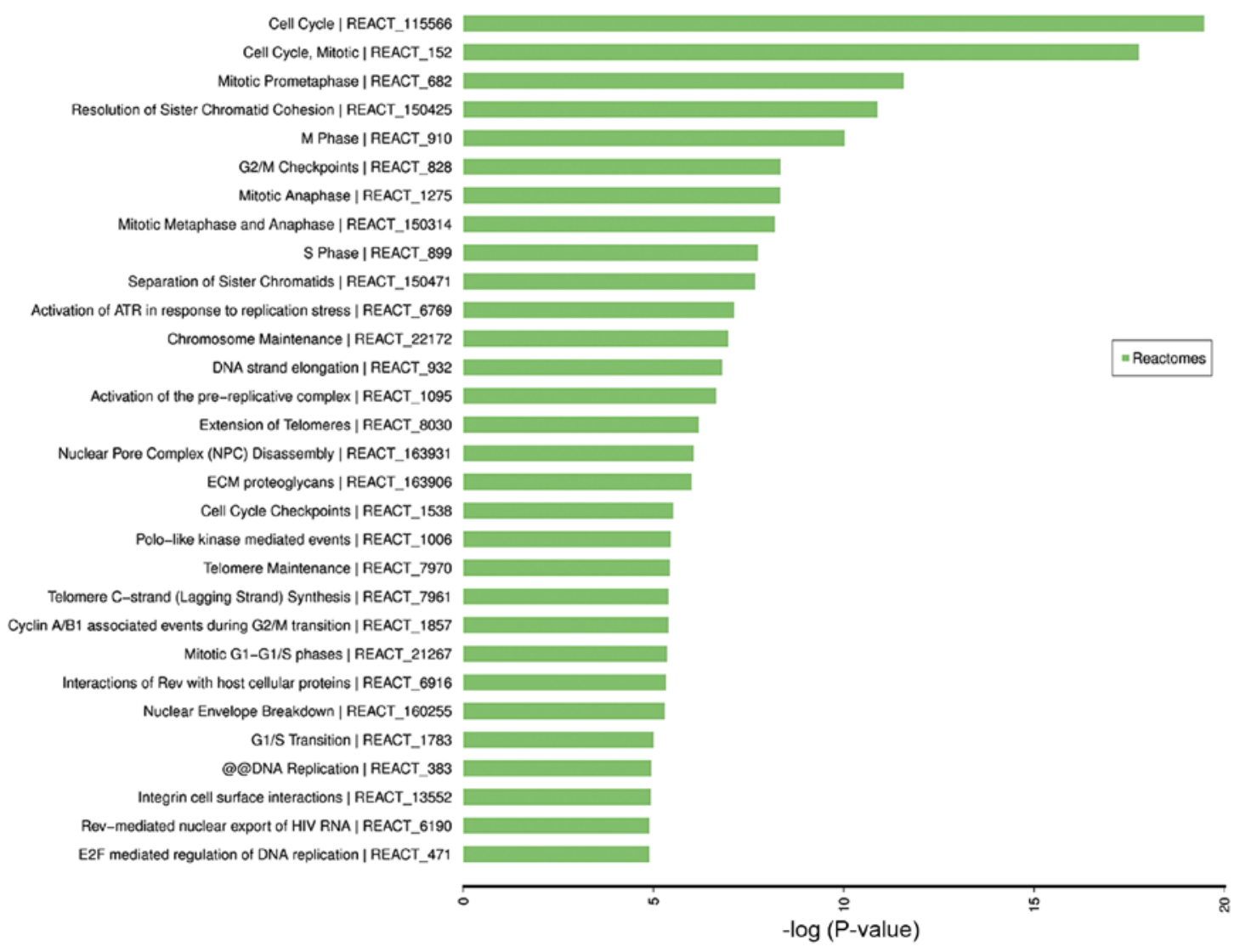

B Significant enriched KEGG PATHWAYS. Pathway terms (top 30)

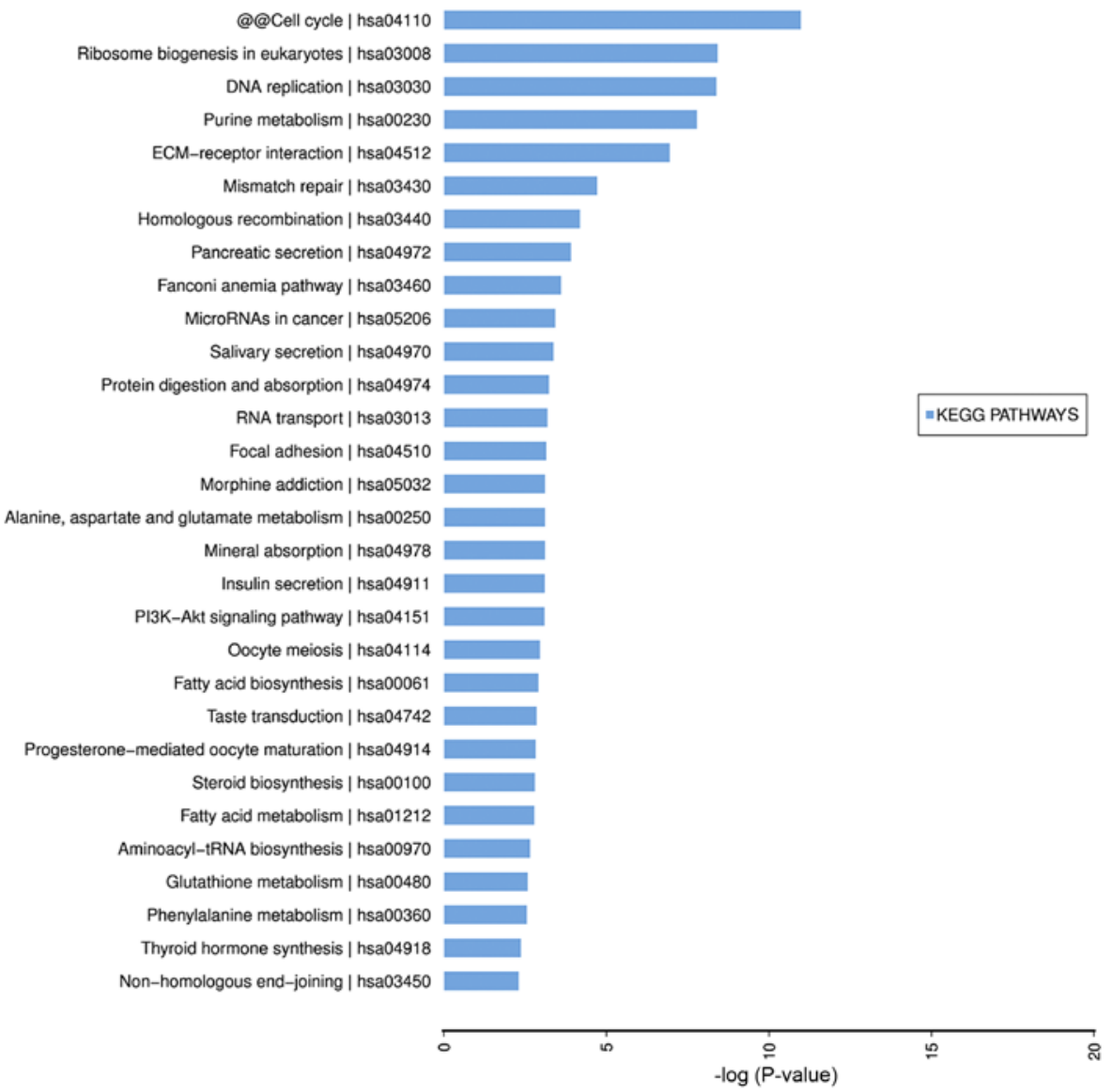

Figure 4. The enriched pathways. The top 30 most significant enriched (A) Reactome pathways and (B) KEGG pathways. 

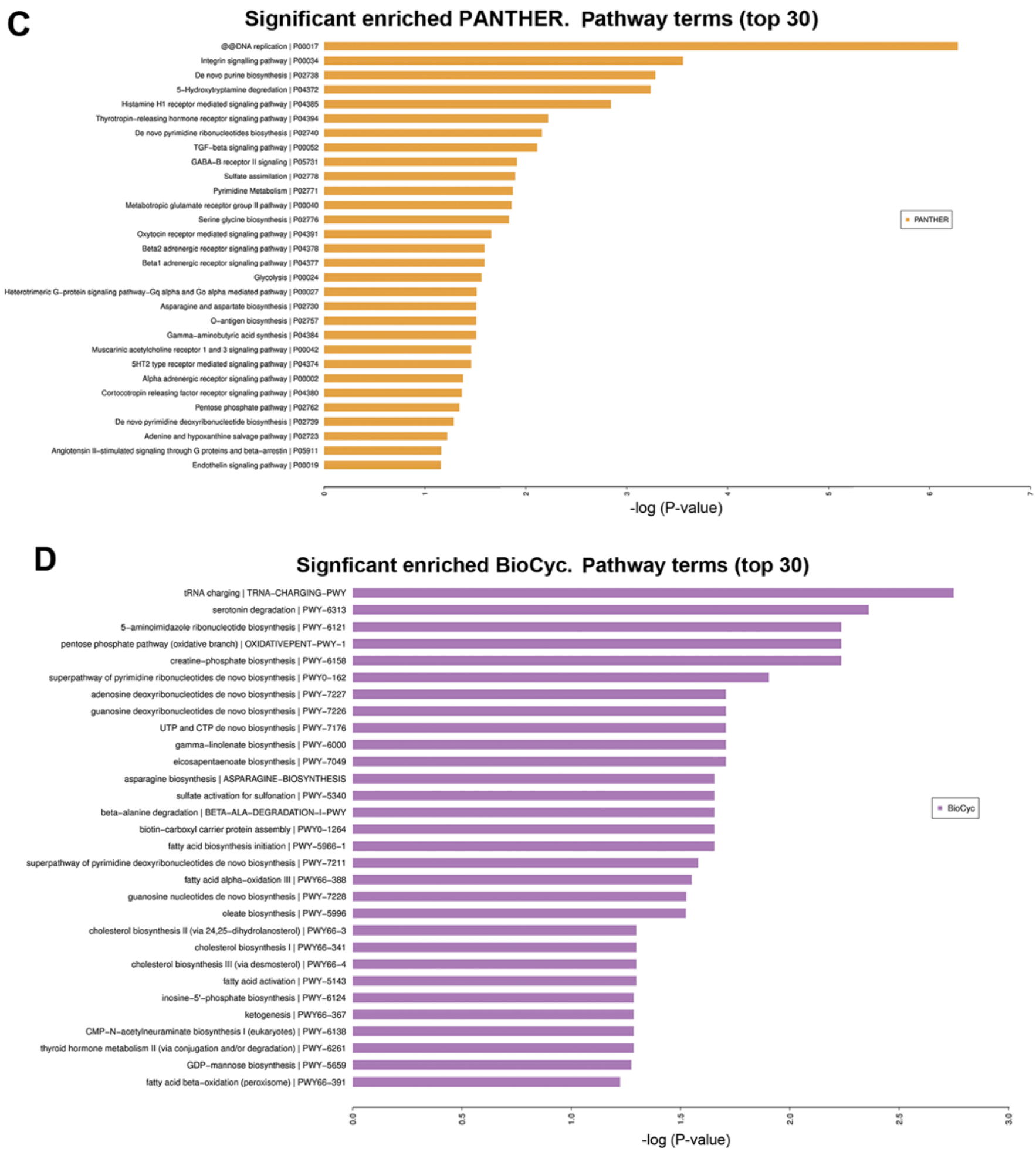

Figure 4. Continued. The top 30 most significant enriched (C) Panther pathways and (D) BioCyc pathways.

phenotype. Gerber et al (22) revealed that SLC4A4 was significantly upregulated in chronic myeloid leukemia (CML) cells and persistent leukemia stem cells, which may be used to develop novel therapies for CML. In the present study, we found that the expression of hsa_circ_0126897_CBCl and its target gene were significantly decreased in CRC tissue (Fig. 6), and that this circRNA participated in many regulated processes of miRNAs. Hence, it was suspected that hsa_circ_0126897_CBCl was a potential biomarker for CRC.
In future, the design of related studies to research the function of $h s a \_c i r c \_0126897 \_C B C l$ in CRC cells and in animal models will be undertaken, to elucidate the related mechanism involved.

In conclusion, $h s a \_c i r c \_0126897 \_C B C 1$ may be a potential biomarker for CRC, and circRNAs could affect the BPs or pathways of the cell cycle thus influencing the occurrence and development of CRC. In addition, fundamental research should focus on circRNAs in the future. 


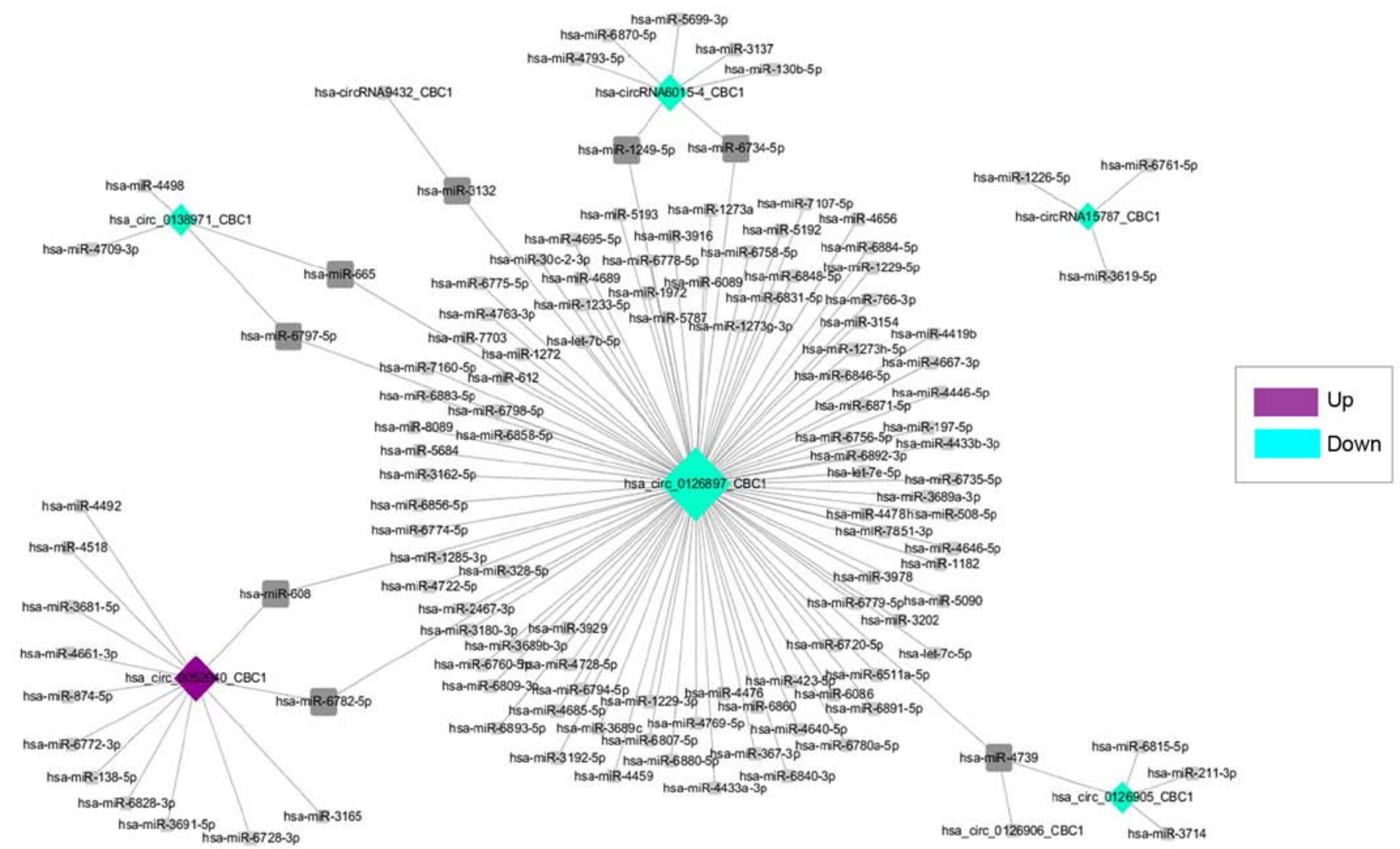

Figure 5. CircRNA/miRNA network. The downregulated differentially expressed circRNAs (green star), the upregulated differentially expressed circRNAs (purple star), and the related miRNAs (gray box). circRNA, circular RNA.

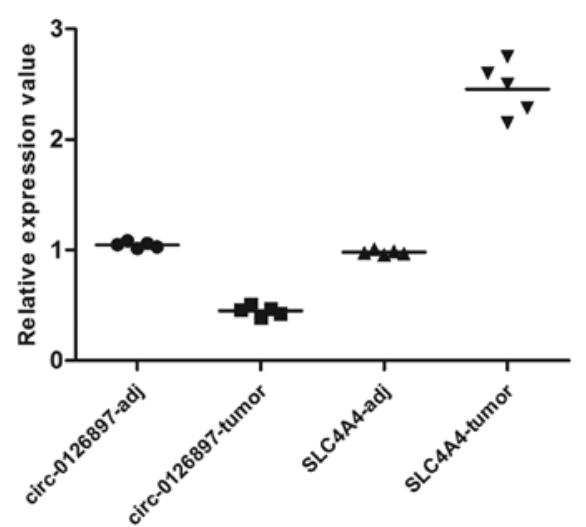

Figure 6. The relative expression value of $h s a_{-} c i r c \_0126897 \_C B C 1$ and SLC4A4 in CRC tissues and their adjacent tissues. CRC, colorectal cancer.

\section{Acknowledgements}

We would like to thank all the members of our research group for their enthusiastic participation in this study.

\section{Funding}

No funding was received.

\section{Availability of data and materials}

The datasets used during the present study are available from the corresponding author upon reasonable request.

\section{Authors' contributions}

XZ designed the experiments. SC and LZ performed data analysis. YS interpretated the data. SC, LZ and YS edited the manuscript. XZ reviewed and edited the manuscript. All authors read and approved the manuscript and agree to be accountable for all aspects of the research in ensuring that the accuracy or integrity of any part of the work are appropriately investigated and resolved.

\section{Ethics approval and consent to participate}

The experimental study was approved by the Ethics Committee of Nankai University Affiliated Hospital, and the informed consent forms were obtained when the patients were accepted for the study by the hospital. All procedures performed in the study involving human participants were in accordance with the ethical standards of the Institutional and/ or National Research Committee and with the 1964 Helsinki declaration and its later amendments or comparable ethical standards.

\section{Consent for publication}

Not applicable.

\section{Competing interests}

The authors declare that they have no competing interests. 


\section{References}

1. Song M, Garrett WS and Chan AT: Nutrients, foods, and colorectal cancer prevention. Gastroenterology 148: 1244-60. e16, 2015.

2. Siegel RL, Miller KD, Fedewa SA, Ahnen DJ, Meester RGS, Barzi A and Jemal A: Colorectal cancer statistics, 2017. CA Cancer J Clin 67: 177-193, 2017.

3. Mäkinen MJ: Colorectal serrated adenocarcinoma. Histopathology 50: 131-150, 2007.

4. Wilusz JE and Sharp PA: Molecular biology. A circuitous route to noncoding RNA. Science 340: 440-441, 2013.

5. Jeck WR, Sorrentino JA, Wang K, Slevin MK, Burd CE, Liu J, Marzluff WF and Sharpless NE: Circular RNAs are abundant, conserved, and associated with ALU repeats. RNA 19: 141-157, 2013.

6. Ding XC, Weiler J and Grosshans H: Regulating the regulators: Mechanisms controlling the maturation of microRNAs. Trends Biotechnol 27: 27-36, 2009.

7. Shannon P, Markiel A, Ozier O, Baliga NS, Wang JT, Ramage D, Amin N, Schwikowski B and Ideker T: Cytoscape: A software environment for integrated models of biomolecular interaction networks. Genome Res 13: 2498-2504, 2003.

8. Taborda MI, Ramírez S and Bernal G; MI T: Circular RNAs in colorectal cancer: Possible roles in regulation of cancer cells. World J Gastrointest Oncol 9: 62-69, 2017.

9. Arvelo F, Sojo F and Cotte C: Biology of colorectal cancer. Ecancermedicalscience 9: 520, 2015.

10. Bachmayr-Heyda A, Reiner AT, Auer K, Sukhbaatar N, Aust S, Bachleitner-Hofmann T, Mesteri I, Grunt TW, Zeillinger R and Pils D: Correlation of circular RNA abundance with proliferation - exemplified with colorectal and ovarian cancer, idiopathic lung fibrosis, and normal human tissues. Sci Rep 5: 8057, 2015.

11. Gillett CE and Barnes DM: Demystified ... cell cycle. Mol Pathol 51: 310-316, 1998.

12. Bartek J and Lukas J: Cell cycle. Order from destruction. Science 294: 66-67, 2001

13. Perez R, Wu N, Klipfel AA and Beart RW Jr: A better cell cycle target for gene therapy of colorectal cancer: Cyclin $\mathrm{G}$. J Gastrointest Surg 7: 884-889, 2003.

14. Du WW, Yang W, Liu E, Yang Z, Dhaliwal P and Yang BB Foxo3 circular RNA retards cell cycle progression via forming ternary complexes with p21 and CDK2. Nucleic Acids Res 44 2846-2858, 2016.
15. Xue J, Liu Y, Luo F, Lu X, Xu H, Liu X, Lu L, Yang Q, Chen C, Fan W, et al: Circ100284, via miR-217 regulation of EZH2, is involved in the arsenite-accelerated cell cycle of human keratinocytes in carcinogenesis. Biochim Biophys Acta 1863: 753-763, 2017.

16. Qin M, Liu G, Huo X, Tao X, Sun X, Ge Z, Yang J, Fan J, Liu L and Qin W: hsa_circ_0001649: A circular RNA and potential novel biomarker for hepatocellular carcinoma. Cancer Biomark 16: 161-169, 2016.

17. Zhao Z, Li X, Gao C, Jian D, Hao P, Rao L and Li M: Peripheral blood circular RNA hsa_circ_0124644 can be used as a diagnostic biomarker of coronary artery disease. Sci Rep 7: 39918, 2017.

18. Cui X, Niu W, Kong L, He M, Jiang K, Chen S, Zhong A, Li W, Lu J and Zhang L: hsa circRNA_103636: Potential novel diagnostic and therapeutic biomarker in Major depressive disorder. Biomarkers Med 10: 943-952, 2016.

19. Chen J, Li Y, Zheng Q, Bao C, He J, Chen B, Lyu D, Zheng B, $\mathrm{Xu}$ Y, Long Z, et al: Circular RNA profile identifies circPVT1 as a proliferative factor and prognostic marker in gastric cancer. Cancer Lett 388: 208-219, 2017.

20. Shang X, Li G, Liu H, Li T, Liu J, Zhao Q and Wang C: Comprehensive circular RNA profiling reveals that hsa circ_0005075, a new circular RNA biomarker, is involved in hepatocellular carcinoma development. Medicine (Baltimore) 95: e3811, 2016.

21. Parks SK and Pouyssegur J: The $\mathrm{Na}(+) / \mathrm{HCO}_{3}(-)$ Co-transporter SLC4A4 Pplays a role in growth and migration of colon and breast cancer cells. J Cell Physiol 230: 1954-1963, 2015.

22. Gerber JM, Gucwa JL, Esopi D, Gurel M, Haffner MC, Vala M, Nelson WG, Jones RJ and Yegnasubramanian S: Genome-wide comparison of the transcriptomes of highly enriched normal and chronic myeloid leukemia stem and progenitor cell populations. Oncotarget 4: 715-728, 2013.

This work is licensed under a Creative Commons Attribution-NonCommercial-NoDerivatives 4.0 International (CC BY-NC-ND 4.0) License. 